\title{
Surrogate Model Assisted Design of CSRR Structure using Genetic Algorithm for Microstrip Antenna Application
}

\author{
Kumaresh SARMAH ${ }^{1,2}$, Sivaranjan GOSWAMI ${ }^{1}$, Sunandan BARUAH ${ }^{2}$ \\ ${ }^{1}$ Dept. of Electronics and Communication Technology, Gauhati University, Guwahati 781014, India \\ 2 Dept. of Electronics and Communication Engineering, Assam Don Bosco University, Guwahati 781017, India \\ kumaresh@gauhati.ac.in,sivgos@gmail.com, sunandan.baruah@dbuniversity.ac.in
}

Submitted March 3, 2019 / Accepted November 25, 2019

\begin{abstract}
Soft-computational approaches have enabled quicker and more efficient means for antenna design. In the present work, a genetic algorithm (GA) based method is reported for the design of complementary split-ring resonator (CSRR) structures for antenna design. A multi-objective optimization problem is formulated to design the antenna. The cost function of the optimization problem is calculated from a surrogate model of the CSRR structure. The surrogate model is created first using an analytical model of the CSRR structure and then using an artificial neural network (ANN). A comparative study of the result shows that the ANN-based surrogate model is accurate as compared to the surrogate model using an analytical approach. An antenna with an integrated filter is fabricated using a CSRR structure designed applying the proposed method. The performance of the antenna is validated from the simulation and measurement results.
\end{abstract}

\section{Keywords}

Artificial neural network, surrogate model, complementary split-ring resonator, microstrip antenna, genetic algorithm, soft-computational design

\section{Introduction}

Multi-objective antenna design is a complex process which involves optimization of the physical dimensions of an antenna to meet multiple desired goals. It is indispensable for a designer to identify the tradeoff between various design goals for this purpose. Soft-computational multiobjective simulations are essential for identifying and optimizing these tradeoffs [1]. In antenna design problems, the three most commonly used soft-computational algorithms are: genetic algorithm (GA) [2], [3], particle swarm optimization (PSO) [4], [5], and differential evolution (DE) [6], [7]. The complementary split-ring resonator (CSRR) structure is a metamaterial structure reported by Falcone et al. as a band-stop filtering element in a microstrip line [8]. This structure yields an effective negative value of the permittivity, $\varepsilon$ of a material at a narrow band near its resonant frequency. Owing to the negative value of permittivity, a microstrip line loaded with a CSRR structure at its ground plane eliminates a narrow band near its resonant frequency. Later, the CSRR structure became popular in the design of microstrip antennas. CSRR structure is reported in the use of dual-band [9-11] and UWB antennas with integrated filter [12], [13]. The CSRR structure is also reported in an antenna for a superheterodyne receiver which has an inherent capability to eliminate two possible image frequencies [14].

A full-wave EM simulation involves a huge set of computations and hence such simulations are often timeconsuming. This significantly limits the efficiency of evolutionary approaches for the design of antennas. A solution to this problem is the introduction of a surrogate model. In [15], a surrogate model assisted evolutionary algorithm (SAEA) is proposed. Surrogate modeling methods include Gaussian process or Kriging, response surface methods, artificial neural networks (ANN), support vector machines and radial basis function models. In [16], a surrogate model-assisted optimization technique for metamaterial devices is proposed. Surrogate models are explored in [17] for constrained, multi-objective antenna design problems.

A surrogate model is a low-fidelity model which is cheap in terms of computational time and resources. Surrogate models are of two types - physics-based surrogate model and approximation-based surrogate model [18]. In a physics-based surrogate model, an analytical expression is derived from the known operating principle of the structure. An approximation-based surrogate model is an approximation of a high fidelity full-wave model which is created by training a soft-computational tool such as an artificial neural network (ANN) with results obtained from the high-fidelity model.

In this paper, a GA-based approach is used to optimize the dimensions of a CSRR structure for the designing of a microstrip antenna with an integrated filter. The CSRR structure has two resonant frequencies where it acts as a notch filtering element owing to the negative value of its 

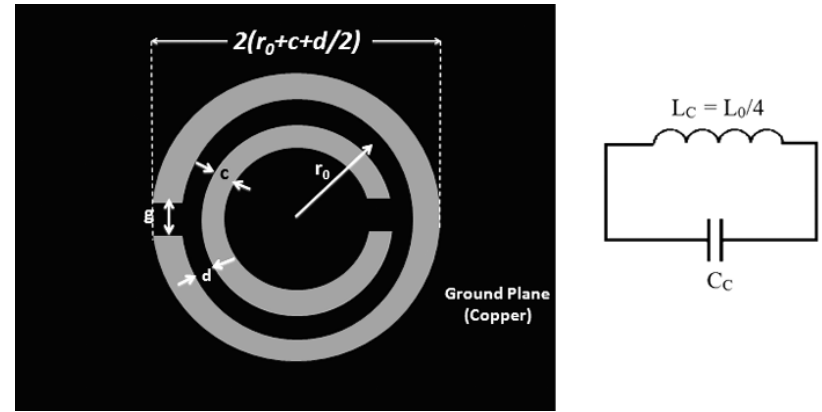

Fig. 1. (a) The topology of the CSRR structure. (b) The LC equivalent circuit of the CSRR structure.

effective permittivity $\left(\varepsilon_{\mathrm{r}}\right)$ [14]. The two resonant frequencies depend upon the dimensions of the CSRR structure. Thus, the design of a CSRR structure may be formulated as a multi-objective optimization problem where the input parameters are the physical dimensions of the CSRR structure as shown in Fig. 1 and the output parameters are the two resonant frequencies of the CSRR structure.

The arrangement of the remaining sections of the paper is as follows. The construction of the physics-based surrogate model and the approximation-based surrogate model are discussed in Sec. 2. The construction of the CSRR structure using GA and the approximation-based surrogate model is discussed in Sec. 3. In Sec. 4, the performance of the two surrogate models is analyzed from experimental results. The paper is concluded in Sec. 5 .

\section{Formulation of the Surrogate Model of the CSRR Structure}

In this section, two surrogate models of the CSRR structure are presented. An existing LC-tank circuit analytical model of the CSRR structure is used as the physicsbased surrogate model. An ANN is trained with results obtained from full-wave simulations to construct the approximation-based surrogate model.

\subsection{The Physics-Based Surrogate Model of the CSRR Structure}

The CSRR structure yields negative value of the permittivity $(\varepsilon)$ at a narrow band near its resonant frequency. This behavior enables the use of CSRR structure as filtering elements at its resonant frequency. The CSRR structure is modeled as an LC tank circuit as shown in Fig. 1. The values of $L_{0}$ and $C_{\mathrm{C}}$ are computed analytically from the physical dimensions of the CSRR structure. The expression for computation of $C_{\mathrm{C}}$ and $L_{0}$ are available in [19] and [20] respectively. These expressions are shown in (1) and (2):

$C_{\mathrm{C}}=$

$\frac{\pi^{3} \varepsilon_{0}}{c^{2}} \int_{0}^{\infty} \frac{[b B(k b)-a B(k a)]^{2}}{k^{2}}\left[0.5\left(1+\frac{1+\frac{\varepsilon}{\varepsilon_{0}} \tanh (k h)}{1+\frac{\varepsilon_{0}}{\varepsilon} \tanh (k h)}\right)\right] \mathrm{d} k$

$$
L_{0}=\frac{\pi^{3} \mu_{0}}{4 c^{2}} \int_{0}^{\infty} \frac{[b B(k b)-a B(k a)]^{2}}{k^{2}} \mathrm{~d} k
$$

Here, $a$ and $b$ are given by (3) and (4) respectively:

$$
\begin{gathered}
a=r_{0}-\frac{c}{2}, \\
b=c+a .
\end{gathered}
$$

$B(x)$ is given by $(5)$

$$
B(x)=S_{0}(x) J_{1}(x)-S_{1}(x) J_{0}(x) .
$$

$S_{n}(x)$ is the $n^{\text {th }}$ order Struve function of $x$ and $J_{n}(x)$ is the $n^{\text {th }}$ order Bessel function of $x$.

The value of $L_{\mathrm{C}}$ can be calculated from $\mathrm{L}_{0}$ using (6)

$$
L_{\mathrm{C}}=\frac{L_{0}}{4} \text {. }
$$

Once, $L_{\mathrm{C}}$ and $C_{\mathrm{C}}$ are calculated, the resonant frequency of the LC-tank circuit can be determined using (7)

$$
f_{\mathrm{r}}=\frac{1}{2 \pi \sqrt{L_{\mathrm{C}} C_{\mathrm{C}}}} .
$$

This set of expressions can be used as a surrogate model to compute the resonant frequency of the CSRR structure.

In practice, a physics-based surrogate model requires further fine-tuning of its parameters which is usually carried out with the help of a high-fidelity model. This increases the accuracy of the low fidelity model.

\subsection{The ANN Based Surrogate Model}

In order to construct the approximation-based surrogate model of the CSRR structure, the first two resonant frequencies of a CSRR structure are obtained experimentally using Ansys HFSS ${ }^{\circledR}$ for several combinations of the geometrical parameters of the CSRR structure. The experimental method used for this measurement is illustrated in [11]. The topology of the neural network used is shown in Fig. 2.

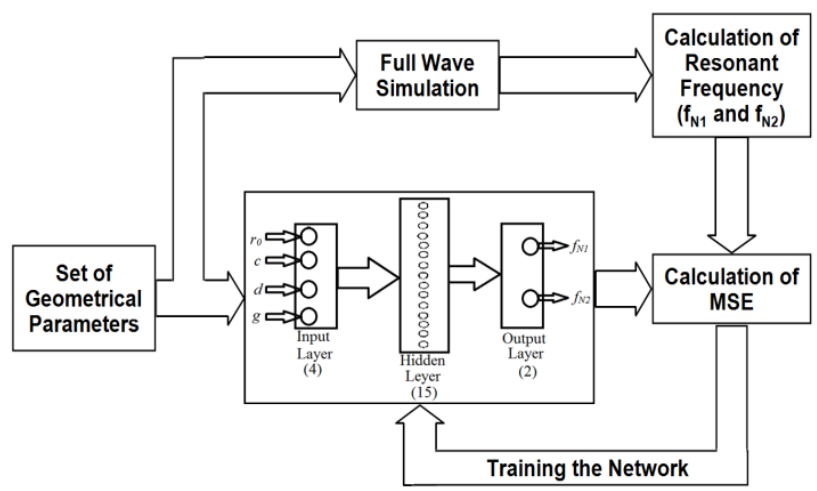

Fig. 2. The structure of the ANN used to create the surrogate model of the CSRR based notch filter. 


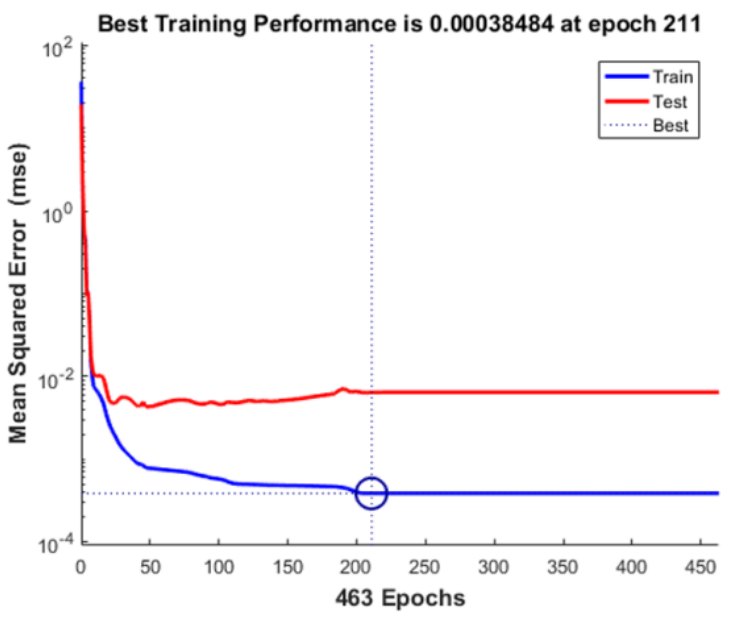

(a)

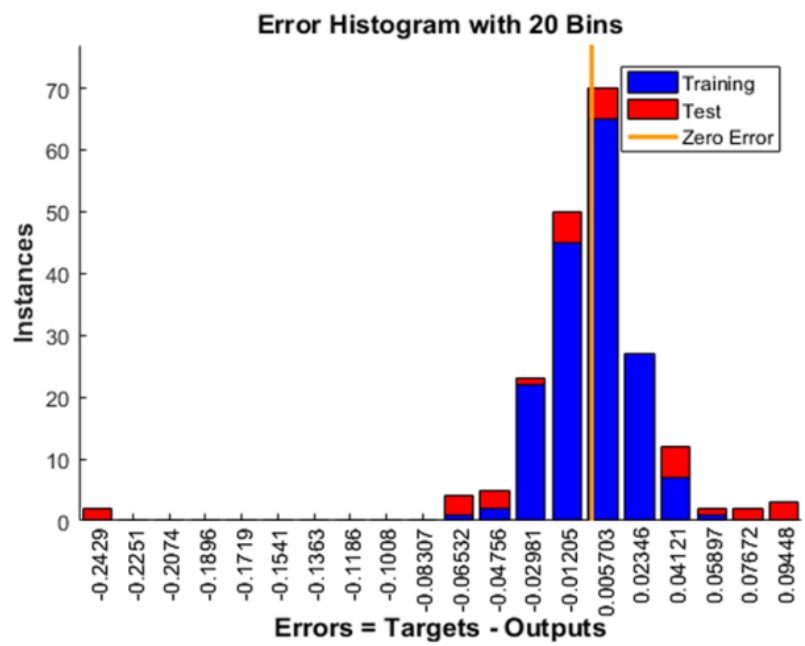

(b)

Fig. 3. (a) Training performance curve. (b) Error histogram of the trained network for the design of CSRR based notch filter.

Bayesian regularization method is used for training the ANN. Bayesian regularized networks require less data for cross-validation and they are robust against overfitting and overtraining [21]. From experimental observations also it is verified that Bayesian regularization as a training function yields better performance of the surrogate model. The number of hidden layers is tuned by repetitive training to maximize the accuracy of the network.

The four nodes at the input layer of the ANN correspond to the four geometrical parameters of the CSRR structure, namely, $r_{0}, c, d$, and $g$. The two output neurons at the output layer correspond to the fundamental resonant frequency of the CSRR structure, $f_{0}$, and its first harmonic, $f_{1}$. The network is trained with a large set of experimental results which comprises of 150 simulation results. 50 more simulation results are used for testing the trained network. The training performance plot and the error histogram of the network are shown in Fig. 3(a) and (b), respectively. The training is performed many times by adjusting the size of the hidden layer. The training time was observed to be in a range of 40-100 seconds. Finally, the number of neurons at the hidden layer is set to 15 , as it provided the minimum error.

\section{The GA Based Design of the CSRR Structure}

GA is used for optimizing the geometrical parameters of a structure to obtain desired performance. The first step of the process is to initialize the population of geometrical parameters of the structure. The performance parameters of the structure $\left(f_{0}\right.$, and $\left.f_{1}\right)$ corresponding to a given set of geometrical parameters $\left(r_{0}, c, d\right.$, and $\left.g\right)$ are estimated from the surrogate model. The cost function taken here is the mean square error (MSE) between the obtained performance parameters of the structure and the desired performance parameters of the structure.

From the discussion in Sec. 2, it is evident that the physics-based surrogate model of the CSRR structure derived from the analytical expressions can estimate only the fundamental resonant frequency of the CSRR structure. The ANN-based surrogate model, on the other hand, is trained for two resonant frequencies of the structure. Thus, for the multi-objective design problem of the CSRR structure, the ANN-based surrogate model is more suitable. Therefore, the ANN-based surrogate model is used with the genetic algorithm to find the performance parameters of the structure from its geometrical parameters.

Genetic algorithm is a direct search algorithm which converges to a minimum value of the cost function. Until any

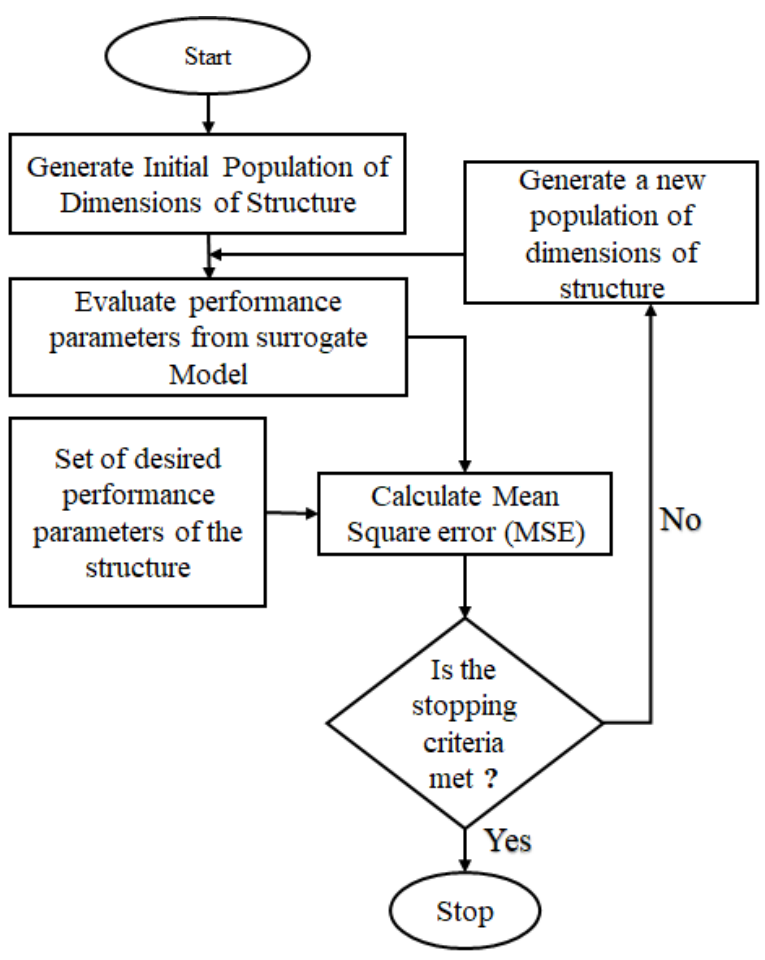

Fig. 4. Optimization algorithm of CSRR structure using Genetic Algorithm. 
of the stopping criteria is met, it iteratively generates a population of the geometrical parameters. Figure 4 shows the flow diagram of the process using a genetic algorithm. Here, the rules of crossover and mutation of the genetic algorithm are used for creating a new population. The use of the surrogate model significantly reduces the computation time as it does not involve actual full-wave simulation of the structure.

\section{Experimental Results and Discussions}

In this section, the two surrogate models are compared based on speed and accuracy. The resonant frequency of the CSRR structure for a set of dimensions is calculated using the analytical model and the ANN model. The accuracy is validated from full-wave analysis using Ansys HFSS $\AA$. Finally, a CSRR structure is designed for a specific antenna application. The designed antenna is tested with a rectangular patch antenna with integrated notch filters. The performance of the antenna is evaluated from simulation and measurement results.

\subsection{Analysis of Computation Time}

The comparative analysis of the time is shown in Tab. 1. The two surrogate models are implemented using Matlab ${ }^{\circledR}$ on a Windows 8.1 operating system on a computer with Intel ${ }^{\circledR} \mathrm{i} 3$ processor and 8 GB RAM. From the table, it is observed that both the analytical model and the ANN-based model are faster than the full-wave analysis performed using Ansys HFSS $\AA$. However, the ANN model is much faster as compared to the analytical model. The involvement of the computationally intensive Struve function

\begin{tabular}{|c|c|c|c|c|c|c|c|}
\hline \multirow{2}{*}{ 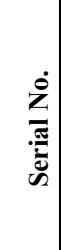 } & \multicolumn{4}{|c|}{$\begin{array}{l}\text { CSRR dimensions } \\
(\mathrm{mm})\end{array}$} & \multicolumn{3}{|c|}{$\begin{array}{l}\text { Computation time } \\
\text { (in seconds) }\end{array}$} \\
\hline & $r_{0}$ & $c$ & $d$ & $g$ & $\begin{array}{l}\infty \\
\hat{\Xi} \\
\end{array}$ & 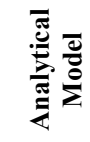 & 玄焉 \\
\hline 1 & 3.50 & 0.50 & 2.00 & 1.00 & 665 & 4.78 & 0.144 \\
\hline 2 & 3.25 & 0.25 & 1.00 & 2.00 & 692 & 8.86 & 0.095 \\
\hline 3 & 4.00 & 0.50 & 1.00 & 1.50 & 458 & 8.79 & 0.281 \\
\hline 4 & 3.75 & 0.25 & 1.00 & 1.00 & 694 & 6.43 & 0.023 \\
\hline 5 & 3.75 & 0.75 & 1.00 & 1.00 & 610 & 8.00 & 0.011 \\
\hline 6 & 3.50 & 1.25 & 1.00 & 1.50 & 449 & 4.71 & 0.011 \\
\hline 7 & 3.50 & 2.00 & 1.00 & 2.00 & 503 & 6.11 & 0.010 \\
\hline 8 & 3.00 & 2.00 & 1.00 & 1.5 & 584 & 8.58 & 0.011 \\
\hline 9 & 4.00 & 2.00 & 0.50 & 1.50 & 708 & 7.96 & 0.010 \\
\hline 10 & 5.00 & 0.50 & 1.00 & 1.00 & 710 & 8.80 & 0.010 \\
\hline
\end{tabular}

Tab. 1. Comparative analysis of the computation time of the analytical model and the ANN-based model of the CSRR structure. and Bessel function makes the analytical surrogate model slow. It is relevant to mention here that almost 2 days were required to simulate 200 randomly picked sets of dimensions of the CSRR structure to train the network. The whole process was performed by writing a python script which tuned the HFSS project variables corresponding to the physical dimensions of the CSRR structure, performed simulations, and stored the results in CSV files. This time is not considered in Tab. 1 as training is a one-time process and once the network is trained, no more simulation is needed.

From Tab. 1, it is observed that the computation time of the analytical model is many times more compared to that of the analytical model. It is because of the fact that the analytical model requires numerical computation of integrals in infinite limit. Standard library routines available in Matlab ${ }^{\circledR}$ are used for this computation.

\subsection{Comparison of the Performance}

The two models are compared in terms of their accuracy. The analytical model of the CSRR structure can estimate only the fundamental resonant frequency of the CSRR structure. The ANN model, on the other hand, is trained to estimate two separate resonant frequencies. Table 2 shows the comparison of the fundamental resonant frequency of the CSRR structure obtained from the analytical model and that by the full-wave simulation using Ansys HFSS $®$. The comparison of the first and the second resonant frequencies of the CSRR structure as obtained from the ANN model with the simulation result are shown in Tab. 3. In Tab. 2 and Tab. 3, only the serial number experiments are given. The physical dimensions $\left(r_{0}, c, d\right.$, and $g$ ) are the same as Tab. 1 .

\begin{tabular}{|c|c|c|c|}
\hline \multirow{2}{*}{ 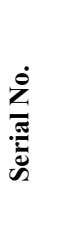 } & \multicolumn{2}{|c|}{$\begin{array}{l}\text { Fundamental resonant } \\
\text { frequency }(\mathrm{GHz})\end{array}$} & \multirow[b]{2}{*}{$\begin{array}{c}\text { Percentage Error } \\
(\%)\end{array}$} \\
\hline & 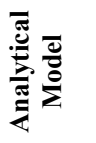 & $\begin{array}{l}\text { Dे } \\
\text { 至 }\end{array}$ & \\
\hline 1 & 3.13 & 3.10 & 0.93 \\
\hline 2 & 3.54 & 3.50 & 1.10 \\
\hline 3 & 2.72 & 2.70 & 0.73 \\
\hline 4 & 3.10 & 3.10 & 0.05 \\
\hline 5 & 2.76 & 2.90 & 4.84 \\
\hline 6 & 2.66 & 2.90 & 8.42 \\
\hline 7 & 2.42 & 2.90 & 16.60 \\
\hline 8 & 2.74 & 3.30 & 17.06 \\
\hline 9 & 2.18 & 2.50 & 12.79 \\
\hline 10 & 2.04 & 2.10 & 2.89 \\
\hline
\end{tabular}

Tab. 2. Comparison of the fundamental resonant frequencies obtained from the analytical model and full-wave simulation using HFSS. 


\begin{tabular}{|c|c|c|c|c|c|c|}
\hline \multirow[b]{2}{*}{$\frac{\dot{0}}{\dot{z}}$} & \multicolumn{3}{|c|}{$\begin{array}{l}\text { Fundamental resonant } \\
\text { frequency }\end{array}$} & \multicolumn{3}{|c|}{$\begin{array}{l}\text { Second resonant } \\
\text { frequency }\end{array}$} \\
\hline & 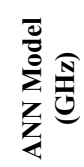 & 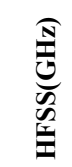 & 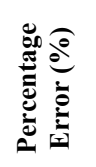 & 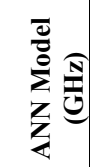 & 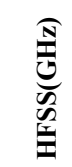 & 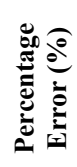 \\
\hline 1 & 3.07 & 3.10 & 1.12 & 7.82 & 7.00 & 11.77 \\
\hline 2 & 3.47 & 3.50 & 0.92 & 6.92 & 6.30 & 9.84 \\
\hline 3 & 2.86 & 2.70 & 5.87 & 5.82 & 5.20 & 11.97 \\
\hline 4 & 3.12 & 3.10 & 0.51 & 5.78 & 5.40 & 7.04 \\
\hline 5 & 2.99 & 2.90 & 3.00 & 6.47 & 5.80 & 11.61 \\
\hline 6 & 3.05 & 2.90 & 5.01 & 7.44 & 6.80 & 9.41 \\
\hline 7 & 2.86 & 2.90 & 1.27 & 7.78 & 7.30 & 6.61 \\
\hline 8 & 3.21 & 3.30 & 2.76 & 9.49 & 9.20 & 3.10 \\
\hline 9 & 2.58 & 2.50 & 3.40 & 5.75 & 5.90 & 2.47 \\
\hline 10 & 2.15 & 2.10 & 2.57 & 4.17 & 4.00 & 4.21 \\
\hline
\end{tabular}

Tab. 3. Comparison of the first and the second resonant frequencies obtained from ANN model and full-wave simulation using HFSS.

From Tab. 2 and Tab. 3 it is observed that the minimum error generated is less in case of the analytical model. However, the ANN model is more consistent. The maximum error is higher in case of the analytical model. Moreover, the ANN model can be trained to estimate two resonant frequencies of the CSRR structure which is not possible in the analytical model.

The performance of the analytical model can be improved further by fine-tuning some of its parameters with the help of data obtained from the HFSS simulation. However, since the model does not predict the second resonant frequency of the CSRR structure, the model will require some major changes.

\subsection{Design of a CSRR Based Antenna using Genetic Algorithm}

Due to the negative value of permittivity, the CSRR structures help in designing antennas with integrated filters. An antenna with a CSRR structure to suppress two possible image frequencies in a superheterodyne receiver was proposed

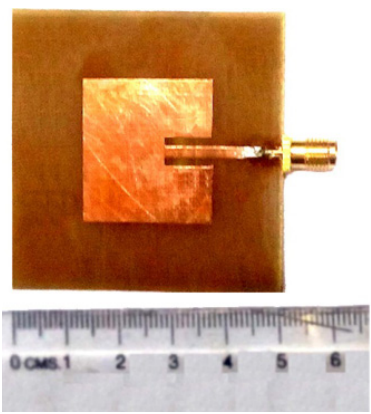

(a)

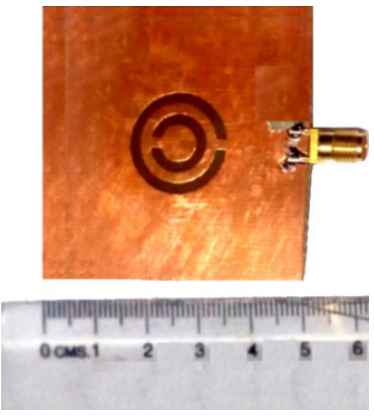

(b)
Fig. 5. (a) Top view of the fabricated antenna and (b) CSRR at its ground plane.

\begin{tabular}{|l|c|}
\hline Geometrical Parameter of the Antenna & Values in (mm) \\
\hline Average radius of the CSRR structure, $r_{0}$ & 6.8064 \\
\hline Width of the rings, $c$ & 1.6126 \\
\hline Gap between the rings, $d$ & 2.5157 \\
\hline Split of the rings, $g$ & 1.5781 \\
\hline Length of the patch, $L$ & 24.2 \\
\hline Width of the patch, $W$ & 26 \\
\hline Width of the feed line & 2 \\
\hline Length of the insets & 11.7 \\
\hline Width of the insets & 1 \\
\hline
\end{tabular}

Tab. 4. Geometrical parameters of the antenna obtained using GA.

in [14]. A GA based optimization technique is validated by designing an antenna with CSRR at its ground plane. Here, the physical dimensions of the CSRR structure are optimized such that the two resonance frequencies are obtained at $1.4 \mathrm{GHz}$ and $3.6 \mathrm{GHz}$. From the above discussion, it is observed that the analytical model of the CSRR structure cannot predict the second resonant frequency of the CSRR structure. Further, the ANN-based model is faster and less prone to errors. Therefore, the ANN-based surrogate model is used in this problem to create the antenna with an integrated filter. The dimensions of the CSRR structure to obtain notch at $1.4 \mathrm{GHz}$ and $3.6 \mathrm{GHz}$ is obtained from the genetic algorithm as shown in Tab. 4. The patch antenna is designed to obtain resonance at $2.4 \mathrm{GHz}$. The length of the patch $(L)$ is $24.2 \mathrm{~mm}$, the width of the patch $(W)$ is $26 \mathrm{~mm}$ and the dielectric substrate used is FR4 epoxy which has a relative permittivity $\left(\varepsilon_{\mathrm{r}}\right)$ of 4.4 .

The fabricated antenna is shown in Fig. 5. The $S_{11}$ parameter of the antenna is measured using a Rohde and Schwarz ${ }^{\circledR}$ ZNB20 VNA. The far-field radiation pattern is measured using the VNA along with a standard horn antenna and with software-controlled automatic turn-table. The simulated and the measured frequency response of the $\mathrm{S}_{11}$ parameter are shown in Fig. 6 . It is observed that the resonant frequency obtained from simulation is $2.4 \mathrm{GHz}$ whereas the measured resonant frequency is $2.36 \mathrm{GHz}$. The two notched frequencies are obtained at $1.35 \mathrm{GHz}$ and 3.65 GHz for the fabricated antenna due to possible imperfections in the fabrication process. It is observed from Fig. 6 that the return loss parameters indicate resonance at $2.4 \mathrm{GHz}$ and near the two-notch frequencies. The actual notch filtering behavior of the antenna is understood from the frequency response of the far-field gain of the antenna along the direction of the major lobe.

The simulated frequency versus gain plot is shown in Fig. 7. It is observed that the gain is the maximum at the resonant frequency of the antenna which is found to be $2.4 \mathrm{GHz}$. At the notch frequencies $1.4 \mathrm{GHz}$ and $3.6 \mathrm{GHz}$, on the other hand, the gain shows significant attenuation. This is due to the negative permittivity of the material as a result of resonance of the CSRR. The normalized radiation pattern of the simulated and fabricated antenna at three different frequencies is shown in Fig. 8. From the radiation pattern plot, it can be further validated that with the optimized dimension of CSRR structure the designed antenna shows significant attenuation at its notched frequencies. Table 5 shows a comparative analysis of the simulated and meas- 


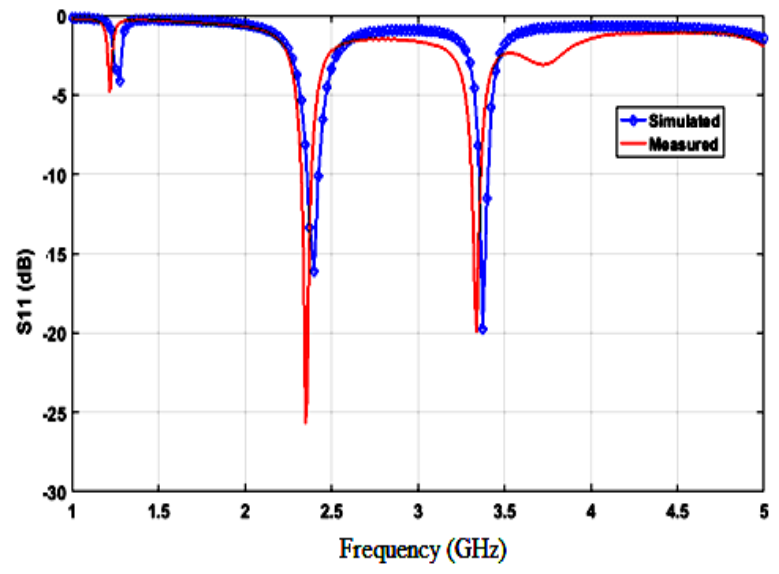

Fig. 6. The simulated and measured frequency response of far-filed radiation pattern of the antenna.

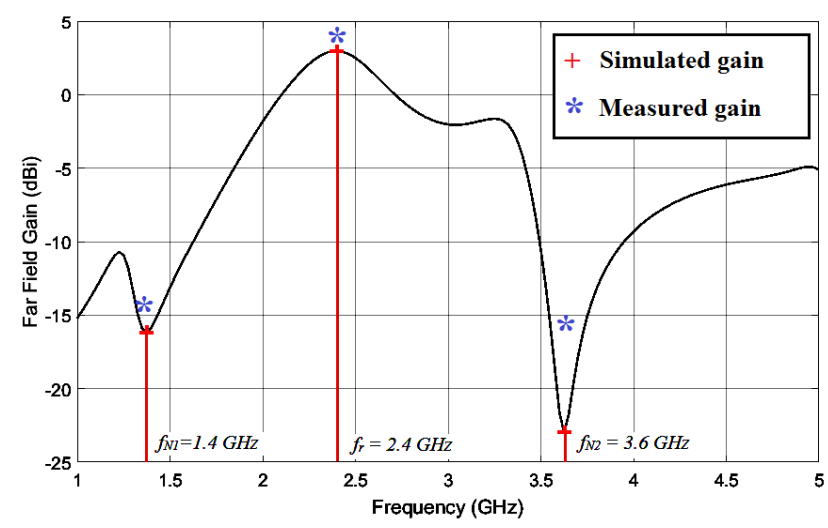

Fig. 7. Simulated frequency vs gain plot of the proposed antenna along the direction of the main lobe indicating two notched frequency.

\begin{tabular}{|c|c|c|}
\hline \multirow{2}{*}{ Frequency $(\mathrm{GHz})$} & \multicolumn{2}{|c|}{ Peak gain of the main lobe $(\mathrm{dB})$} \\
\cline { 2 - 3 } & Simulated & Measured \\
\hline 1.4 & -16.3 & -13.9 \\
\hline 2.4 & 2.1 & 3.2 \\
\hline 3.6 & -23.5 & -15.8 \\
\hline
\end{tabular}

Tab. 5. Comparison of measured and simulated gains of the antenna at the operating frequency and the notch frequencies.

ured gains of the antenna at the operating frequency and at the notch frequencies.

\subsection{Analysis of the Fabrication Tolerance}

The GA based design of the CSRR structure provides a dimension of the CSRR structure with a precision of $0.0001 \mathrm{~mm}$. However, it is practically not feasible to build an antenna with such a high precision. Therefore, it is necessary to provide an analysis of the fabrication tolerance of the antenna. Figure 9 and Figure 10 compares the simulated frequency responses of the $S_{11}$ parameter and the far field gain for a precision of $0.0001 \mathrm{~mm}$ and a precision of $0.1 \mathrm{~mm}$. From these results, it is evident that the proposed antenna has a high fabrication tolerance.

From Fig. 9 and Fig. 10 it can be seen that for the resonant frequency of the antenna at $2.4 \mathrm{GHz}$, there is no

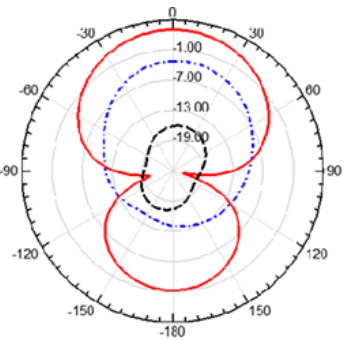

(a) Azimuthal Plane (Simulated)

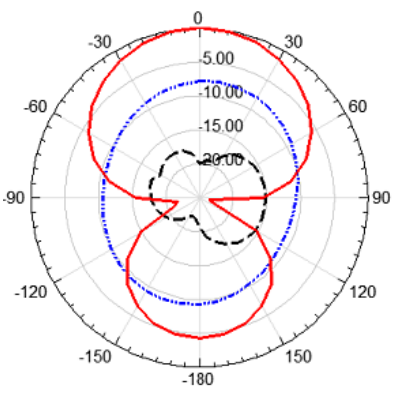

(c) Azimuthal Plane (Measured)

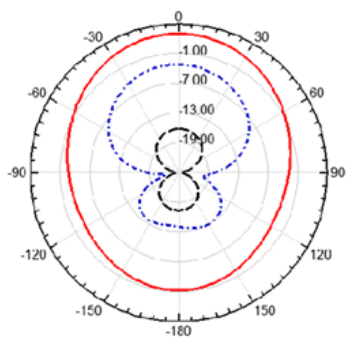

(b) Elevation Plane (Simulated)

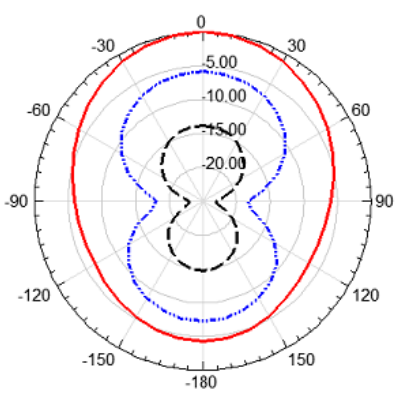

(d) Elivation Plane (Measured)

$$
\begin{array}{r}
f_{r}=2.4 \mathrm{GHz} \\
f_{N 1}=1.4 \mathrm{GHz} \\
-f_{N 2}=3.6 \mathrm{GHz}
\end{array}
$$

Fig. 8. Simulated and measured far field radiation pattern of the proposed antenna at the three frequencies in azimuthal plane and elevation plane.

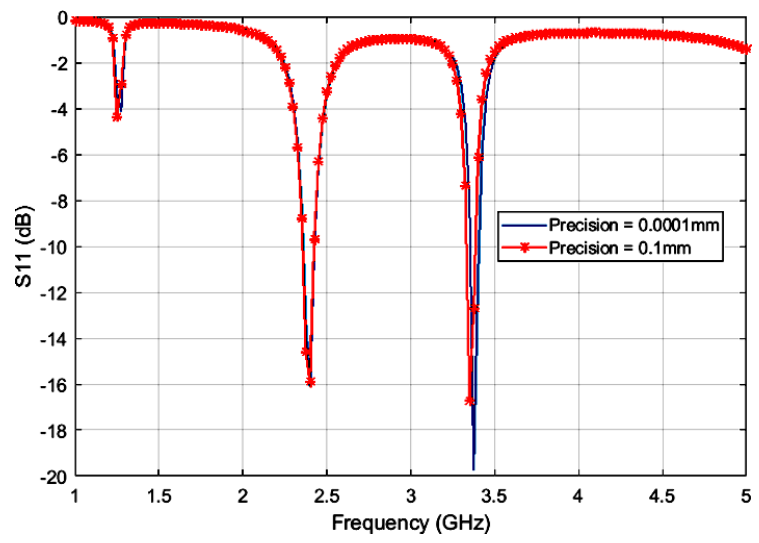

Fig. 9. Comparison of the S11 parameter plots of the designed antenna for a fabrication precision of $0.0001 \mathrm{~mm}$ and $0.1 \mathrm{~mm}$.

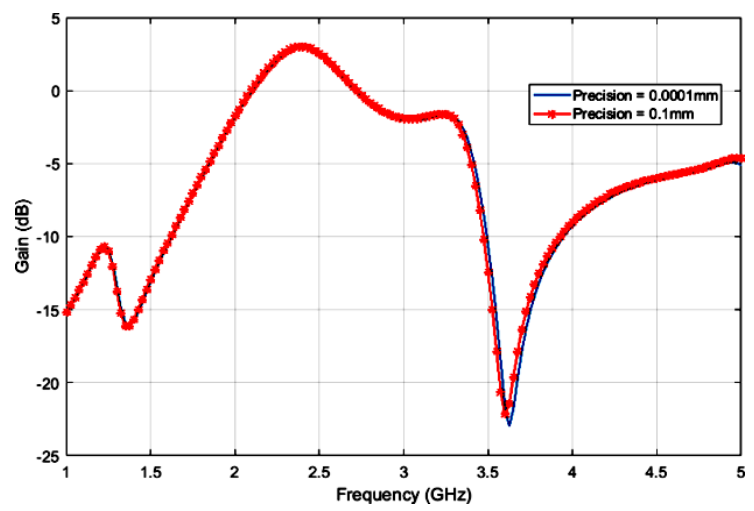

Fig. 10. Comparison of the far-field gain plots of the designed antenna for a fabrication precision of $0.0001 \mathrm{~mm}$ and $0.1 \mathrm{~mm}$. 
change in the performance of the antenna when precision is reduced to $0.1 \mathrm{~mm}$. There is a negligibly small shift in the upper notch band of the antenna.

\subsection{Discussion}

It can be inferred that the ANN-based surrogate model is more accurate and faster compared to the traditional analytical model of the CSRR structure. The ANN-based model can be trained with two resonant frequencies of the structure. The only limitation of the ANN-based model is the requirement of a large dataset for training the network. The performance of the network must be maximized by selecting the proper training function and the size of the hidden layer. The suitability of the proposed method is validated by designing a CSRR structure for an antenna with an integrated dual-band notch filter.

\section{Conclusion}

A surrogate model-assisted soft-computational design method for the CSRR structure is proposed in this paper. The surrogate model is created using a conventional analytical model and an ANN-based model. It is found that the ANN-based model is more persistent and less prone to errors. Further, the ANN-based model required lesser computation time and it can be trained to estimate more than one resonant frequency. The proposed method is validated by comparing with results obtained from full-wave simulation using Ansys HFSS $®$.

The proposed method is further validated by designing a CSRR structure for a microstrip antenna for a superheterodyne receiver which resonates at $2.4 \mathrm{GHz}$ and has the inherent capacity to notch two possible image frequencies at $1.4 \mathrm{GHz}$ and $3.6 \mathrm{GHz}$. The performance of the CSRR loaded antenna is evaluated from simulation and measurement results.

\section{Acknowledgments}

The authors would like to thank for the research facilities provided by Gauhati University and Assam Don Bosco University to carry out the experimental and measurement works.

\section{References}

[1] DEB, K. Multi-Objective Optimization Using Evolutionary Algorithms: An Introduction. New York (USA): John Wiley \& Sons, 2001. ISBN: 047187339X

[2] BHATTACHARYA, S., CHATTOPADHYAY, S., TALUKDER, S., et al. Optimization of inset-fed microstrip patch antenna using genetic algorithm. In The proceedings of the International Conference and Workshop on Computing and Communication (IEMCON). Vancouver (BC, Canada), 2015, p. 1-4. DOI: 10.1109/IEMCON.2015.7344525
[3] LAMSAlli, M., El HAMiCHI, A., BOUSSOUIS, M., et al. Genetic algorithm optimization for microstrip patch antenna miniaturization. Progress In Electromagnetics Research Letters, 2016, vol. 60, p. 113-120. DOI: 10.2528/PIERL16041907

[4] WANG, N. Z., WANG, X. B., XU, J. D. Design of a novel compact broadband patch antenna using binary PSO. Microwave and Optical Technology Letters, 2012, vol. 54, no. 2, p. 434-438. DOI: $10.1002 / \mathrm{mop} .26552$

[5] PRATAP, P., BHATIA, R. S., KUMAR, B. Design and simulation of equilateral triangular microstrip antenna using particle swarm optimization (PSO) and advanced particle swarm optimization (APSO). Sadhana, 2016, vol. 41, no. 7, p. 721-725. DOI: 10.1007/s12046-016-0510-y

[6] GOUdOS, S. K., SIAKAVARA, K., SAMARAS, T., et al. Selfadaptive differential evolution applied to real-valued antenna and microwave design problems. IEEE Transactions on Antennas and Propagation, 2011, vol. 59, no. 4, p. 1286-1298. DOI: 10.1109/TAP.2011.2109678

[7] GANGOPADHYAYA, M., MUKHERJEe, P., SHARMA, U., et al. Design optimization of microstrip fed rectangular microstrip antenna using differential evolution algorithm. In The 2nd IEEE International Conference on Recent Trends in Information Systems (ReTIS). Kolkata (India), 2015, p. 49-52. DOI: 10.1109/ReTIS.2015.7232851

[8] FAlCONe, F., LOPETEGI, T., BAENA, J. D., et al. Effective negative $-\varepsilon$ stopband microstrip lines based on complementary split ring resonators. IEEE Microwave and Wireless Components Letters, 2004, vol. 14, no. 6, p. 280-282. DOI: 10.1109/LMWC.2004.828029

[9] SARMAH, K., GOSWAMI, S., SARMA, A., et al. An experimental study on designing of a dual band antenna using CSRR structure with a single band antenna. In The Proceedings of the 2nd IEEE International Conference on Computational Electromagnetics (ICCEM). Guangzhou (China), 2016, p. 141-143. DOI: 10.1109/COMPEM.2016.7588563

[10] SARKAR, D., SAURAV, K., SRIVASTAVA, K. V. Design of a novel dual-band microstrip patch antenna for WLAN/WiMAX applications using complementary split ring resonators and partially defected ground structure. In Progress in Electromagnetic Research Symposium Proceedings. Taipei (Taiwan), 2013, p. 821-825. ISBN: 978-1-934142-24-0

[11] SARMAH, K., GOSWAMI, S., SARMA, K. K., et al. Design of a CSRR based dual band rectangular patch antenna with predictable far field radiation pattern. In Proceedings of the $6^{\text {th }}$ International Conference on Computers and Devices for Communication (CODEC). Kolkata (India), 2015, p. 1-4. ISBN: $978-1-4673-9513-7 / 15$

[12] KIM, D. O., JO, N. I., JANG, H. A., et al. Design of the ultrawideband antenna with a quadruple-band rejection characteristics using a combination of the complementary split ring resonators. Progress In Electromagnetics Research, 2011, vol. 112, p. 93-107. DOI: 10.2528/PIER10111607

[13] ZHANG, Y., HONG, W., YU, C., et al. Planar ultrawideband antennas with multiple notched bands based on etched slots on the patch and/or split ring resonators on the feed line. IEEE Transactions on Antennas and Propagation, 2008, vol. 56, no. 9, p. 3063-3068. DOI: 10.1109/TAP.2008.928815

[14] GOSWAMI, S., SARMAH, K., SARMA, A., et al. Design of a CSRR based compact microstrip antenna for image rejection in $\mathrm{RF}$ down-converter based WLAN receivers. AEU International Journal of Electronics and Communications, 2017, vol. 74, p. 128-134. DOI: $10.1016 /$ j.aeue.2017.02.004

[15] LIU, B., ALIAKBARIAN, H., MA, Z., et al. An efficient method for antenna design optimization based on evolutionary computation and machine learning techniques. IEEE Transactions on Antennas and Propagation, 2014, vol. 62, no. 1, p. 7-18. DOI: 10.1109/TAP.2013.2283605 
[16] TENUTI, L., SAluCCI, M., OLIVERI, G., et al. Surrogateassisted optimization of metamaterial devices for advanced antenna systems. In Proceedings of IEEE Symposium Series on Computational Intelligence. Cape Town (South Africa), 2015, p. 1154-1156. DOI: 10.1109/SSCI.2015.165

[17] SINGH, P., ROSSI, M., COUCKUYT, I., et al. Constrained multiobjective antenna design optimization using surrogates. International Journal of Numerical Modelling: Electronic Networks, Devices and Fields, 2016, vol. 30, no. 6, p. 1-5. DOI: $10.1002 /$ jnm. 2248

[18] KOZIEL, S., OGURTSOV S. Antenna Design by SimulationDriven Optimization. New York (USA): Springer, 2014. ISBN: 978-3-319-04367-8

[19] BAENA, J. D., BONACHE, J., MARTIN, F., et al. Equivalentcircuit models for split-ring resonators and complementary splitring resonators coupled to planar transmission lines. IEEE Transactions on Microwave Theory and Techniques, 2005, vol. 53, no. 4, p. 1451-1461. DOI: 10.1109/TMTT.2005.845211

[20] MARQUES, R., MESA, F., MARTEL, J., et al. Comparative analysis of edge- and broadside- coupled split ring resonators for metamaterial design - theory and experiments. IEEE Transactions on Antennas and Propagation, 2003, vol. 51, no. 10, p. 2572-2581. DOI: 10.1109 /TAP.2003.817562

[21] BURDEN, F., WINKLER, D. Bayesian regularization of neural networks. Methods in Molecular Biology, 2008, vol. 458, p. 23-42. DOI: 10.1007/978-1-60327-101-1 3

\section{About the Authors ...}

Kumaresh SARMAH (corresponding author) was born in Assam, India in 1982. He received his M.Sc. and M.Phil. degree in Electronics and Communication Technology from Gauhati University in 2006 and 2009, respectively.
He also received M.Tech. degree in Electronics Design and Technology from Tezpur University in 2011. As an Assistant Professor, he joined the Department of Electronics and Communication Technology, Gauhati University in 2013. Currently he is pursuing his Ph.D. degree from Assam Don Bosco University. His research interests include metamaterial and nanomaterial-based antenna design, soft computing and communication.

Sivaranjan GOSWAMI was born in Assam, India in 1991. He received his B.Tech. degree in Electronics and Communication Engineering from Assam Don Bosco University in 2013 and M.Tech. degree in Signal Processing and Communication from Gauhati University in 2015. As an Assistant Professor, he joined the Department of Electronics and Communication Technology, Gauhati University in 2016. His research interests include antenna design, soft computing and signal processing.

Sunandan BARUAH was born in Assam, India in 1971. He holds a Doctor of Engineering degree in Nanotechnology from the Asian Institute of Technology, Thailand. He has a Master of Engineering degree in Microelectronics, also from the Asian Institute of Technology, Thailand, and a Bachelor of Engineering degree in Electronics and Telecommunication from Assam Engineering College, Gauhati University, India. He held teaching and research positions in the Asian Institute of Technology, Thailand and Uppsala University, Sweden. He is currently the Director of the Center of Excellence in Nanotechnology at Assam Don Bosco University. His research interest is in the synthesis, characterization and applications of nanomaterials for applications like antenna design, sensors, energy harvesting, and optical filters etc. 